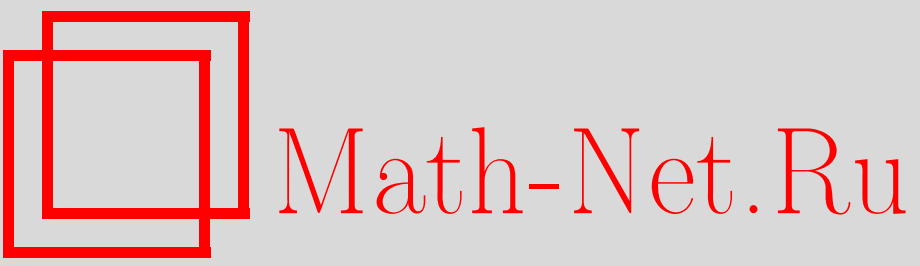

А. Ю. Бондаренко, А. И. Лиходед, В. В. Сидоров, Построение механических аналогов подконструкций с учетом действующих на них активных сил, Maтем. моделирование, 2020, том 32, номер 8, 106-118

DOI: https://doi.org/10.20948/mm-2020-08-07

Использование Общероссийского математического портала Math-Net.Ru подразумевает, что вы прочитали и согласны с пользовательским соглашением

http://www.mathnet.ru/rus/agreement

Параметры загрузки:

IP: 34.227 .88 .159

26 апреля 2023 г., 14:01:45 


\title{
ПОСТРОЕНИЕ МЕХАНИЧЕСКИХ АНАЛОГОВ ПОДКОНСТРУКЦИЙ С УЧЕТОМ ДЕЙСТВУЮЩИХ НА НИХ АКТИВНЫХ СИЛ
}

\author{
(C) 2020 2. $\quad$ А.Н. Бондаренко ${ }^{1,2}$, А.И. Лиходед ${ }^{1,2}$, В.В. Сидоров \\ ${ }^{1}$ Московский физико-технический институт (национальный исследователь- \\ ский университет) \\ ${ }^{2}$ Центральный научно-исследовательский институт машиностроения \\ andrei.bondarenko@phystech.edu
}

DOI: $10.20948 / \mathrm{mm}-2020-08-07$

Рассматривается задача моделирования гидроупругих колебаний жидкого топлива в баках ракет-носителей с использованием механических аналогов. При этом важно обеспечить адекватность расчетной модели с механическими аналогами реальному объекту по массово-инерционным и динамическим характеристикам. Кроме того, необходимо обеспечить корректное задание внешних активных сил, действующих на конструкцию бака, при его моделировании механическими аналогами. Предложенный подход может быть применен при моделировании огневых стендовых испытаний двигательных установок, когда двигатель крепится на ферменной конструкции к днищу бака, а также при анализе нагрузок на перспективные многоразовые ступени ракет-носителей.

Ключевые слова: динамическая задача, расчет нагрузок, эквивалентная балочная модель, механические аналоги, гидроупругие тона колебаний, учет активных сил.

\section{MODELING OF A SPACE-ROCKET STRUCTURES WHEN SUBJECTED TO ACTIVE FORCES BY MECHANICAL ANALOGS}

\author{
A.Yu. Bondarenko ${ }^{1,2}$, A.I. Likhoded ${ }^{1,2}$, V.V.Sidorov ${ }^{2}$ \\ ${ }^{1}$ Moscow Institute of Physics and Technology (National research university) \\ ${ }^{2}$ FSUE the Central scientific research institute for machine building (TSNIIMASH)
}

The problem of modeling hydro-elastic longitudinal oscillations in liquid fuel tanks by mechanical analogs is considered. It is necessary to provide the inertia and dynamic properties coincidence of a real structure and its computational model with mechanical analogs. Special attention has been payed to correct modeling of the external active forces, acting on the fuel tank with engine, when mechanical analogs are used. The proposed approach can be used in modeling of a ground static firing test when engine is mounted on the tank dome, and in dynamic load analysis of the advanced launch-vehicle reusable stages. 
Keywords: dynamic response, load analysis, equivalent beam-like model, mechanical analogs, hydro-elastic modes, active forces.

\section{1. Введение}

В ракетно-космической технике механические аналоги широко применяются для конденсации сложных пространственных подконструкций с целью включения их в упрощенные балочные модели конструкций [1-4 и др.]. Механические аналоги используются для моделирования динамического поведения сложных систем и анализа нагрузок на такие системы, а также анализа динамики и устойчивости движения ракет-носителей (РН) [5-9 и др.].

В частности, включение в динамические модели механических аналогов применяется для учета волновых и гидроупругих колебаний жидкого топлива в баках РН при расчете нагрузок. Поверхностные волновые движения топлива при поперечных колебаниях РН, как правило, слабо влияют на нагрузки. Вместе с тем, для корректности они также могут быть учтены путем включения в динамическую модель соответствующих осцилляторов или маятников [10-15].

Гидроупругие колебания топливных баков существенно влияют на продольные нагрузки РН, поэтому они обязательно должны учитываться в расчетах динамических нагрузок при включении и выключении двигательных установок (ДУ). При конечноэлементном (КЭ) моделировании для учета жидкого топлива может применяться метод виртуальных масс, использующий потенциал простого слоя, встроенный в программный комплекс MSC Nastran. Этот подход позволяет с хорошей точностью описать гидроупругие колебания баков с жидкостью [16-18].

В этой статье описывается способ построения расчетной модели подсистемы с использованием механических аналогов и корректного приложения к ней внешних активных сил. В частности, подобная ситуация возникает при построении механических аналогов топливных баков, к нижнему днищу которых через ферменную конструкцию и шпангоут присоединена двигательная установка (активной силой является тяга).

Для моделирования динамических процессов при запуске и выключении ДУ в штатных и нештатных ситуациях с использованием механических аналогов топливных баков необходимо обеспечить:

- эквивалентность расчетной модели с механическими аналогами по массово-инерционным и динамическим характеристикам;

- корректность задания внешних активных сил на набор аналогов (осцилляторов), моделирующих динамику подконструкции. 


\section{2. Определение величин активных сил, действующих на подсистемы}

Рассмотрим свободную осесимметричную подконструкцию, заданную конечно-элементной моделью

$$
M \tilde{\ddot{X}}+K \tilde{X}=f(t) .
$$

Здесь $M$ и $K$ - матрицы масс и жесткостей, $f(t)$ - вектор внешних сил, $\tilde{X}=\left(\tilde{x}_{1}, \tilde{x}_{2}, \ldots, \tilde{x}_{n}\right)^{T}-$ вектор обобщенных координат. Индекс $T$ означает транспонирование.

Будем считать, что подконструкция возбуждается через плоское балочное сечение, перпендикулярное оси симметрии, по закону $\tilde{x}_{1}=u_{0}(t)$. Представим решение уравнения (1) в виде

$$
X=\tilde{X}-e u_{0}(t),
$$

где $e$ - вектор, компонентами которого являются нули и единицы. Единицы соответствуют обобщенным координатам, описывающим осевое перемещение подконструкции, нули - остальным координатам вектора $\tilde{X}$. Подставляя (2) в (1), получим:

$$
M \ddot{X}+K X=-M e \ddot{u}_{0}+f(t) .
$$

По существу, уравнение (3) описывает динамику осесимметричной системы в подвижной системе координат, которая плоскопараллельно смещается вдоль оси симметрии системы по закону $u_{0}(t)$. При этом выражение - Мeї 0 в правой части уравнения (3) представляет собой осевые инерционные силы. Очевидно, что с учетом (2) обобщенная координата $x_{1}$ вектора $X$, соответствующая $\tilde{x}_{1}$ вектора $\tilde{X}$, равна нулю.

Поэтому решение уравнений (3) будем искать с использованием собственных форм колебаний $\varphi_{j}$ подконструкции, консольно закрепленной в этом сечении. Воспользовавшись разложением вектора $X$ по собственным тонам колебаний консольно закрепленной подконструкции $\varphi_{j}$

$$
X(t)=\sum_{j} \varphi_{j} T_{j}(t),
$$

и обобщенной процедурой Галеркина $[7,19]$, получим следующую систему уравнений относительно обобщенных координат $T_{j}$ :

$$
\varphi_{j}^{T} M \varphi_{j} \ddot{T}_{j}+\varphi_{j}^{T} M \varphi_{j} \omega_{j}^{2} T_{j}=-\varphi_{j}^{T} M e \ddot{u}_{0}+\varphi_{j}^{T} f(t) .
$$


Очевидно, уравнение (5) аналогично уравнению осциллятора, возбуждаемого за точку подвеса в направлении оси $z$ по закону $u_{0}(t)$

$$
m_{j} \ddot{z}+k_{j} z=-m_{j} \ddot{u}_{0}+F_{j}(t)
$$

где $m_{j}$ и $k_{j}$ - масса и жесткость осциллятора, $z$ - растяжение пружины, $F_{j}(t)-$ внешняя сила, действующая на осциллятор (см. рис.1).

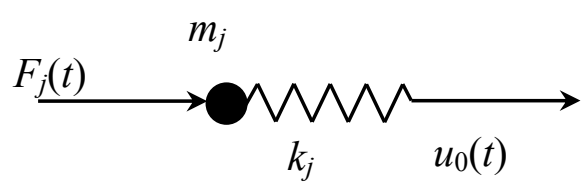

Рис.1. Осциллятор, возбуждаемый за точку подвеса.

Для полной адекватности уравнений (5) для обобщенных координат $T_{j}$ и уравнения для осциллятора (6) необходимо приравнять их коэффициенты:

$$
m_{j}=\varphi_{j}^{T} M \varphi_{j}, \quad k_{j}=\omega_{j}^{2} \varphi_{j}^{T} M \varphi_{j}, \quad m_{j}=\varphi_{j}^{T} M e, \quad F_{j}(t)=\varphi_{j}^{T} f(t) .
$$

Из анализа соотношений (7) следует необходимость обеспечить равенство правых частей первого и третьего равенств:

$$
\varphi_{j}^{T} M \varphi_{j}=\varphi_{j}^{T} M e .
$$

Равенство (8) является ключевым для однозначного определения параметров механических аналогов; его можно обеспечить с учетом того, что собственные формы колебаний определяются с точностью до константы в виде множителя:

$$
\varphi_{j}=C_{j} \tilde{\varphi}_{j}
$$

Поэтому, введя новые формы $\tilde{\varphi}_{j}$ в виде (9), будем иметь

$$
C_{j}=\tilde{\varphi}_{j}^{T} M e /\left(\tilde{\varphi}_{j}^{T} M \tilde{\varphi}_{j}\right) .
$$

Тогда выражения (7) для массы осциллятора $m_{j}$, жесткости $k_{j}$ и силы $F_{j}(t)$ с учетом (8), (10) примут вид

$$
m_{j}=\frac{\left(\tilde{\varphi}_{j}^{T} M e\right)^{2}}{\tilde{\varphi}_{j}^{T} M \tilde{\varphi}_{j}}, \quad k_{j}=m_{j} \omega_{j}^{2}, \quad F_{j}(t)=\frac{\tilde{\varphi}_{j}^{T} M e}{\tilde{\varphi}_{j}^{T} M \tilde{\varphi}_{j}} \tilde{\varphi}_{j}^{T} f(t) .
$$

Формулы (11) упрощаются, если в качестве базисных форм колебаний использовать нормированные по единичной массе формы $\tilde{\varphi}_{j}$ : 


$$
m_{j}=\left(\tilde{\varphi}_{j}^{T} M e\right)^{2}, \quad k_{j}=m_{j} \omega_{j}^{2}, \quad F_{j}(t)=\left(\tilde{\varphi}_{j}^{T} M e\right) \tilde{\varphi}_{j}^{T} f(t) .
$$

Можно показать, что сумма масс осцилляторов стремится к физической массе объекта [20].

Покажем, что сумма обобщенных сил, действующих на осцилляторы, равна физической силе, действующей на конденсируемый объект в осевом направлении. Для этого представим разложение вектора $e^{T}$ в виде обобщенного ряда Фурье по собственным формам колебаний $\tilde{\varphi}_{j}$ подсистемы:

$$
e^{T}=\sum_{j}\left(\tilde{\varphi}_{j}^{T} M e\right) \tilde{\varphi}_{j}^{T}
$$

Умножив справа обе части (13) на вектор внешних осевых сил $f(t)$, приложенный к конденсируемому объекту, получим следующее скалярное равенство:

$$
e^{T} f(t)=\sum_{j}\left(\tilde{\varphi}_{j}^{T} M e\right) \tilde{\varphi}_{j}^{T} f(t) .
$$

Скалярная величина в левой части равенства (14) в соответствии со структурой вектора $e$ представляет собой сумму всех внешних сил $F^{\mathrm{EXT}}$, действующих на подсистему в осевом направлении. Скалярная величина в правой части (14) в соответствии с третьей формулой (12) является суммой обобщенных сил $F_{j}(t)$, действующих на осцилляторы. Таким образом, подтверждается равенство суммы обобщенных сил, приложенных к осцилляторам, суммарной силе, действующей на подсистему в осевом направлении:

$$
F^{E X T}(t)=\sum_{j} F_{j}
$$

Представляет интерес формирование обобщенных сил $F_{j}(t)$ для осцилляторов, моделирующих колебания баков с топливом в тех случаях, когда двигатель крепится через ферменную конструкцию и шпангоут к нижнему днищу бака (см. рис.2). Как правило, продольные гидроупругие (ГУ) колебания топливных баков моделируются несколькими осцилляторами, соответствующими низшим парциальным тонам колебаний бака с жидкостью, закрепленными за нижний шпангоут.

Параметры этих осцилляторов определяются первыми двумя формулами в (12). Учет трех осцилляторов обычно обеспечивает сходимость массовых характеристик топливных баков порядка 85-90\% от их физической массы. Недостающая масса бака компенсируется присоединенной массой в зоне нижнего шпангоута. 


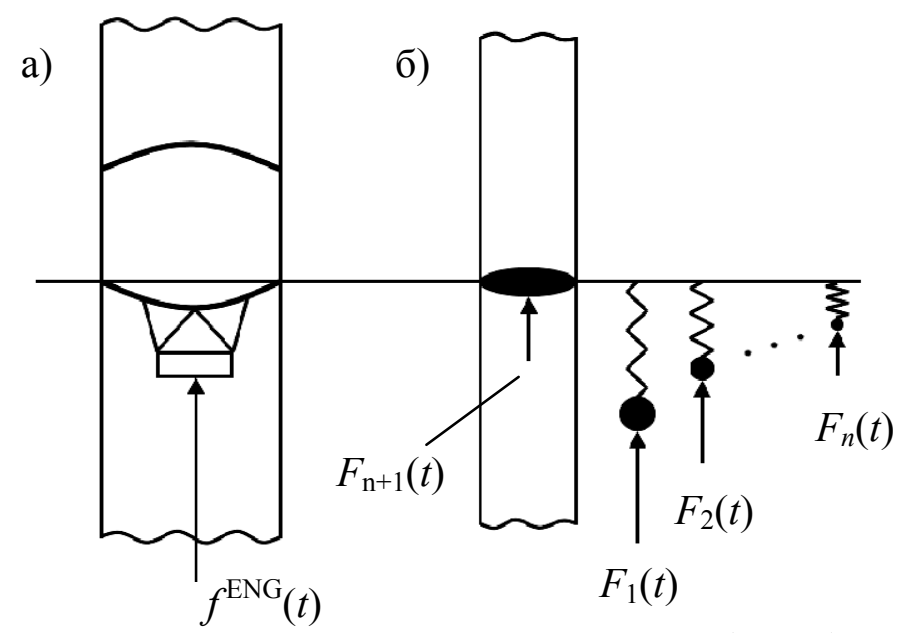

Рис.2. Топливный бак с присоединенным двигателем (слева) и моделирующие его механические аналоги и приведенные силы (справа).

В случае топливного бака с присоединенным к его днищу двигателем (рис.2а) при действии только осевой силы тяги $f^{\mathrm{ENG}}(t)$ от ДУ обобщенные силы, действующие на осцилляторы, в соответствии с (12) примут вид

$$
F_{j}(t)=\left(\tilde{\varphi}_{j}^{T} M e\right) \tilde{\varphi}_{j}^{T} f(t)=\left(\tilde{\varphi}_{j}^{T} M e\right) \tilde{\varphi}_{j}^{\mathrm{ENG}} f^{\mathrm{ENG}}(t),
$$

где $\tilde{\varphi}_{j}^{\mathrm{ENG}}$ - осевое перемещение двигателя по $j$-й форме парциальных колебаний топливного бака с закрепленным на нем двигателем.

Входящее в (16) произведение $\tilde{\varphi}_{j}^{T} M е$ в соответствии с первым равенством (12) связано с массой соответствующего осциллятора:

$$
\left|\left(\tilde{\varphi}_{j}^{T} M e\right)\right|=\sqrt{m_{j}}
$$

Таким образом, обобщённые силы $F_{j}(t)$, действующие на осцилляторы (см. рис.2б), примут следующий окончательный вид:

$$
F_{j}(t)=f^{\mathrm{ENG}}(t) \cdot \lambda_{j}, \quad j=1,2, \ldots, n,
$$

где $n$ - число учитываемых собственных форм колебаний, а $\lambda_{j}-$ безразмерные коэффициенты:

$$
\lambda_{j}=\left(\tilde{\varphi}_{j}^{T} M e\right) \cdot \tilde{\varphi}_{j}^{\mathrm{ENG}} .
$$

Более нагляден тот факт, что коэффициенты $\lambda_{j}$ являются безразмерными, следует из формулы (11), в которой используются ненормированные по единичной массе формы колебаний.

Из равенства (15) с учетом (18), (19) вытекает очевидное равенство для коэффициентов $\lambda_{j}$ 


$$
\sum_{j}\left(\tilde{\varphi}_{j}^{T} M e\right) \cdot \tilde{\varphi}_{j}^{\mathrm{ENG}}=\sum_{j} \lambda_{j}=1 .
$$

Если сумма коэффициентов $\lambda_{1}, \lambda_{2}, \ldots, \lambda_{n}$ приближается к единице, недостающая часть силы

$$
F_{n+1}(t)=f^{\mathrm{ENG}}(t) \cdot \lambda_{n+1}, \quad \lambda_{n+1}=1-\sum_{j=1}^{n} \lambda_{j}
$$

должна быть приложена к нижнему шпангоуту бака.

Таким образом, получены формульные выражения для учета активных сил, действующих на подсистемы, моделируемые механическими аналогами.

\section{3. Валидация предложенной методики}

Для проверки полученных выражений была разработана КЭ-модель типового бака РН, к юбке нижнего днища которого на ферме прикреплен двигатель. Расчеты проводились с использованием программного комплекса MSC Nastran. Днища бака полусферические, между ними гладкая цилиндрическая обечайка. К стыку нижнего днища с обечайкой присоединен промежуточный отсек. ДУ в модели представлена в виде сосредоточенной массы и моментов инерции. Одним из наиболее эффективных методов моделирования жидкого топлива является метод виртуальных масс, встроенный в MSC Nastran [21]. Масса топлива составляла 11 тонн, сухая масса конструкции $\sim 1$ тонна. Было разработано три КЭ-модели, в которых жесткость фермы ДУ варьировалась таким образом, что:

1) частота продольного тона подвески ДУ примерно совпадает с частотой первого гидроупругого тона бака с топливом (далее - «модель 1»);

2) частота продольного тона подвески ДУ лежит между частотами первого и второго тона гидроупругих колебаний бака (далее - «модель 2»);

3) частота продольного тона подвески ДУ выше частот первых трех гидроупругих тонов колебаний бака (далее - «модель $3 »)$.

Внешний вид КЭ-модели бака показан на рис.3.

Общее число смоченных элементов в модели составило $\sim 1200$, всего в модели присутствует порядка 2000 элементов и 1650 узлов. Такая КЭ-схематизация обеспечивает хорошее качество расчетов при небольших затратах компьютерных ресурсов [22].

Для всех моделей был проведен расчет собственных частот и форм колебаний в частотном диапазоне до 150 Гц. Граничные условия соответствовали закреплению центрального узла шпангоута промежуточного отсека по шести степеням свободы. Центральный узел связан со шпангоутом при помощи жесткого элемента (RBE2). 


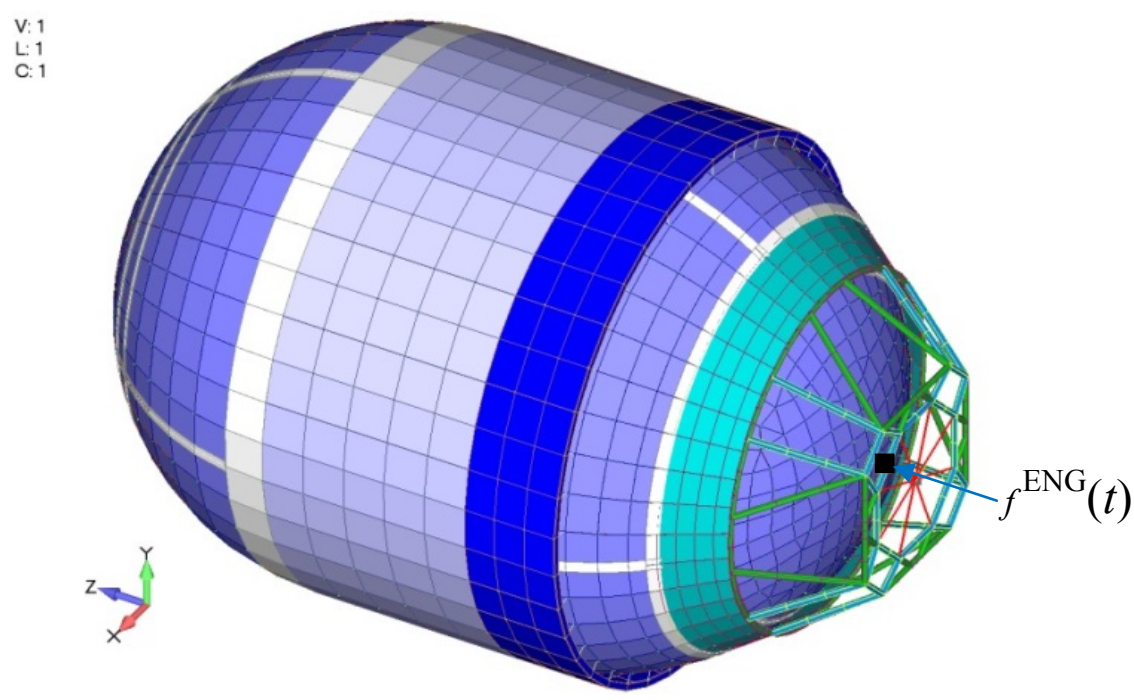

Рис.3. КЭ-модель топливного бака с присоединенным ДУ.

По результатам расчетов для всех моделей были проверены:

- сходимость суммы коэффициентов $\lambda_{j}$ к единице (21);

- совпадение модуля величины $\tilde{\varphi}_{j}^{T} M e$ с корнем из рассчитанной эффективной массы тонов с точностью до знака (18).

Определение величин $\tilde{\varphi}_{j}^{T} M е$ было встроено в последовательность расчета собственных тонов колебаний, для чего разработан модуль на языке DMAP. Результаты расчетов, параметры эквивалентных осцилляторов и коэффициенты $\lambda_{j}$ на силовое воздействие $f^{\mathrm{ENG}}(t)$ приведены в табл.1 (приведены тона с большой осевой эффективной массой).

Из табл. 1 видно, что квадрат величины $\tilde{\varphi}_{j}^{T} M e$ (4-й столбец) совпадает с эффективной массой $m_{j}$ соответствующего тона (5-й столбец).

При выборе достаточного числа тонов сумма коэффициентов $\lambda_{j}$ сходится к единице. Однако эти коэффициенты могут быть отрицательными, они не пропорциональны эффективной массе тонов и их сходимость не связана со сходимостью по эффективной массе. Например, в модели 3 сумма коэффициентов по первым трем гидроупругим тонам, имеющим суммарную эффективную массу более 95\%, составляет 0.55. А величины коэффициентов $\lambda_{5}$ и $\lambda_{82}$ близки, хотя эффективная масса соответствующих тонов различается более чем на два порядка.

На основании табл.1 были разработаны КЭ-модели для моделирования топливного бака с использованием механических аналогов. В каждой эквивалентной модели к закрепленному по всем степеням интерфейсному узлу присоединяется несколько осцилляторов с осевой жесткостью $k_{j}$ и массой $m_{j}$, в каждый из которых приложена сила $\lambda_{j} \cdot f^{\mathrm{ENG}}(t)$. Недостающая часть 
силы приложена в узел интерфейса. Для модели 3 было сделано две эквивалентные модели, в одной из которых учтено только два первых осциллятоpa, а во второй - восемь. Для оценки адекватности моделей была решена следующая задача.

Таблица 1. Параметры эквивалентных продольных осцилляторов и коэффициенты $\lambda_{j}$

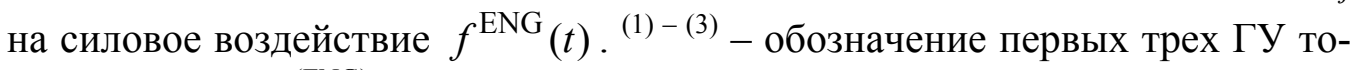
нов колебаний, ${ }^{(E N G)}$ - обозначение тона подвески ДУ. В модели 3 тон ДУ нельзя выделить точно, т.к. он смешивается с ГУ-тонами.

\begin{tabular}{|c|c|c|c|c|c|c|c|c|}
\hline \multicolumn{9}{|c|}{ Модель 1} \\
\hline № & $f, \Gamma ц$ & $\left(\tilde{\varphi}_{j}^{T} M e\right)$ & $\left(\tilde{\varphi}_{j}^{T} M e\right)^{2}$ & $m_{j}$, кг & $m_{j}, \%$ & $\tilde{\varphi}_{j}^{\mathrm{ENG}}$ & $k_{j}, \mathrm{H} / \mathrm{M}$ & $\lambda_{j}$ \\
\hline $5^{(1)}$ & 32.82 & -95.4863 & 9117.62 & 9117.62 & 76.65 & $-2.0955 \mathrm{E}-2$ & $3.877 \mathrm{E}+8$ & 2.000925 \\
\hline $10^{(\mathrm{ENG})}$ & 34.07 & -21.9725 & 482.79 & 482.79 & 4.06 & $3.8481 \mathrm{E}-2$ & $2.213 \mathrm{E}+7$ & -0.845528 \\
\hline $35^{(2)}$ & 61.29 & -36.4134 & 1325.93 & 1325.93 & 11.15 & $5.2629 \mathrm{E}-3$ & $1.966 \mathrm{E}+8$ & -0.191639 \\
\hline $40^{(3)}$ & 82.65 & 24.5859 & 604.47 & 604.47 & 5.08 & $1.2210 \mathrm{E}-3$ & $1.630 \mathrm{E}+8$ & 0.030020 \\
\hline & & & \multicolumn{2}{|c|}{$100-\sum m_{j}, \%$} & 3.06 & & $1-\sum \lambda_{j}$ & 0.006222 \\
\hline \multicolumn{9}{|c|}{ Модель 2} \\
\hline № & $f, \Gamma ц$ & $\left(\tilde{\varphi}_{j}^{T} M e\right)$ & $\left(\tilde{\varphi}_{j}^{T} M e\right)^{2}$ & $m_{j}$, кг & $m_{j}, \%$ & $\tilde{\varphi}_{j}^{\mathrm{ENG}}$ & $k_{j}, \mathrm{H} / \mathrm{M}$ & $\lambda_{j}$ \\
\hline $5^{(1)}$ & 33.04 & 96.6420 & 9339.67 & 9339.67 & 78.51 & $4.4267 \mathrm{E}-3$ & $4.025 \mathrm{E}+8$ & 0.427809 \\
\hline $20^{(\mathrm{ENG})}$ & 39.63 & -18.9114 & 357.64 & 357.64 & 3.01 & $-4.3085 \mathrm{E}-2$ & $2.217 \mathrm{E}+7$ & 0.814799 \\
\hline $35^{(2)}$ & 62.95 & -34.7431 & 1207.08 & 1207.08 & 10.15 & $8.5406 \mathrm{E}-3$ & $1.888 \mathrm{E}+8$ & -0.296726 \\
\hline \multirow[t]{2}{*}{$40^{(3)}$} & 82.74 & 24.5227 & 601.36 & 601.36 & 5.06 & $1.8993 \mathrm{E}-3$ & $1.625 \mathrm{E}+8$ & 0.046577 \\
\hline & & & \multicolumn{2}{|c|}{$100-\sum m_{j}, \%$} & 3.27 & & $1-\sum \lambda_{j}$ & 0.007541 \\
\hline \multicolumn{9}{|c|}{ Модель 3} \\
\hline № & $f, \Gamma ц$ & $\left(\tilde{\varphi}_{j}^{T} M e\right)$ & $\left(\tilde{\varphi}_{j}^{T} M e\right)^{2}$ & $m_{j}$, кг & $m_{j}, \%$ & $\tilde{\varphi}_{j}^{\mathrm{ENG}}$ & $k_{j}, \mathrm{H} / \mathrm{M}$ & $\lambda_{j}$ \\
\hline $5^{(1)}$ & 33.07 & 95.4825 & 9116.91 & 9116.91 & 76.64 & $1.5758 \mathrm{E}-3$ & $3.937 \mathrm{E}+8$ & 0.150460 \\
\hline $30^{(2)}$ & 57.56 & 41.1706 & 1695.02 & 1695.02 & 14.25 & $1.5472 \mathrm{E}-2$ & $2.217 \mathrm{E}+8$ & 0.636981 \\
\hline $36^{(3)}$ & 81.35 & -24.6895 & 609.57 & 609.57 & 5.12 & $9.5047 \mathrm{E}-3$ & $1.593 \mathrm{E}+8$ & -0.234667 \\
\hline 64 & 100.83 & 6.9373 & 48.13 & 48.13 & 0.40 & $5.4932 \mathrm{E}-3$ & $1.931 \mathrm{E}+7$ & 0.038108 \\
\hline 69 & 105.04 & -2.9116 & 8.48 & 8.48 & 0.07 & $-2.2248 \mathrm{E}-2$ & $3.693 \mathrm{E}+6$ & 0.064776 \\
\hline 82 & 122.27 & 5.5098 & 30.36 & 30.36 & 0.26 & $2.2340 \mathrm{E}-2$ & $1.792 \mathrm{E}+7$ & 0.123090 \\
\hline 91 & 127.65 & 7.2094 & 51.98 & 51.98 & 0.44 & $1.7306 \mathrm{E}-2$ & $3.343 \mathrm{E}+7$ & 0.124766 \\
\hline \multirow[t]{2}{*}{105} & 138.79 & 9.2662 & 85.86 & 85.86 & 0.72 & $7.8064 \mathrm{E}-3$ & $6.529 \mathrm{E}+7$ & 0.072336 \\
\hline & & & \multicolumn{2}{|c|}{$100-\sum m_{j}, \%$} & 2.10 & & $1-\sum \lambda_{j}$ & 0.024151 \\
\hline
\end{tabular}

Для каждой из трех полных КЭ-моделей был проведен расчет усилий в заделке (узел в центре шпангоута промежуточного отсека) во временной области при спаде тяги ДУ с некоторого уровня до нуля по закону $f^{\mathrm{ENG}}(t)$. В качестве закона спада тяги была выбрана реальная циклограмма выключения ДУ. Такая постановка задачи соответствует огневым стендовым испытаниям (ОСИ) двигателя. Были также определены усилия в заделке по моделям с механическими аналогами и проведено сравнение с результатами, полученными по полным конечно-элементным моделям. 
На рис.4 сверху приведено сравнение усилия в заделке, определенного по полной модели бака и модели с механическими аналогами, для моделей 1 и 2. На рис. 4 снизу приведено сравнение усилий, рассчитанных по полной модели 3 бака и моделям с двумя и восемью механическими аналогами.

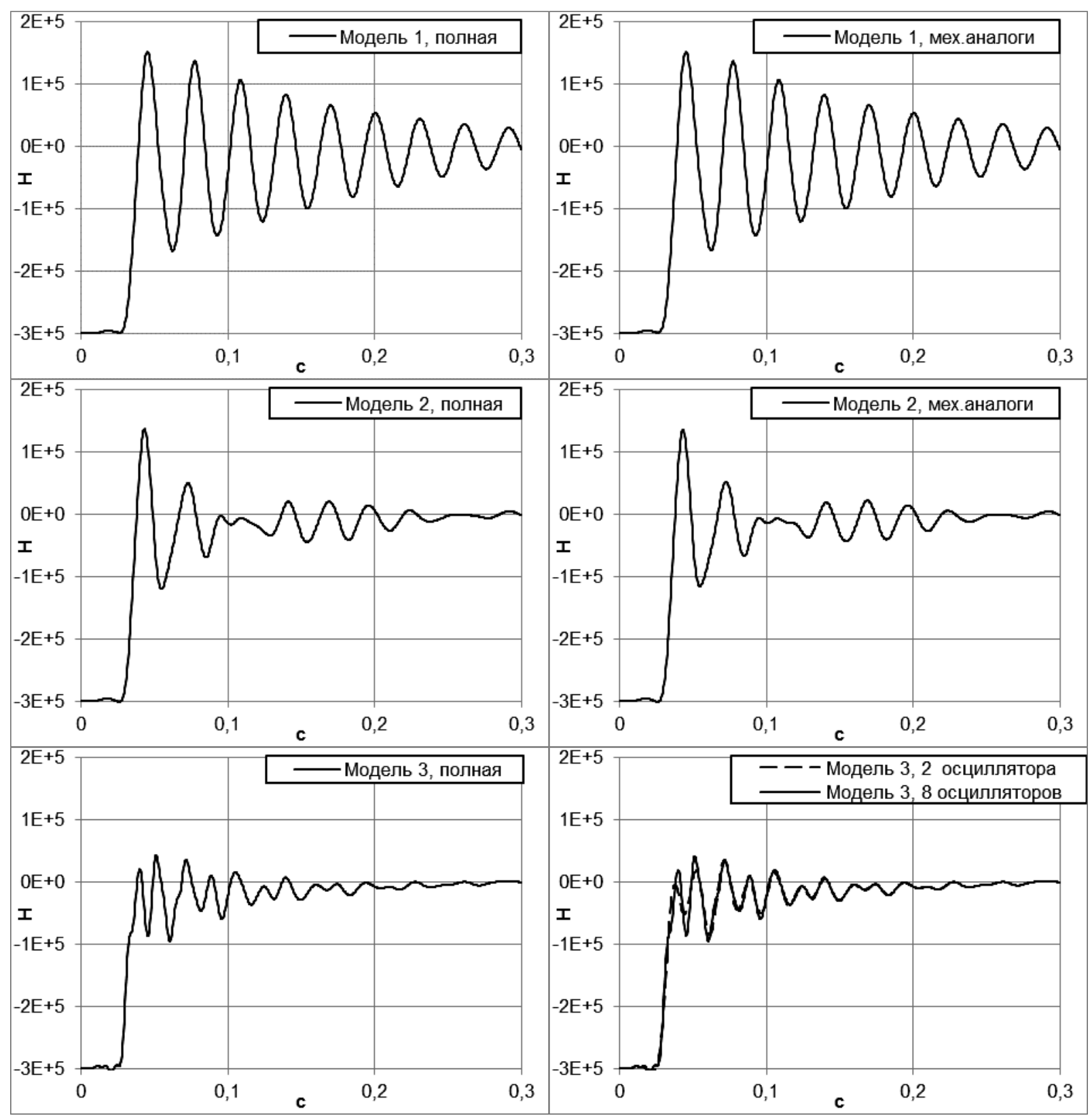

Рис.4. Сравнение усилий в заделке по полным моделям и моделям, содержащим механические аналоги. Сверху - для моделей 1 и 2. Снизу - для модели 3.

Также были рассчитаны усилия в заделке по моделям, включающим механические аналоги, при задании коэффициентов на силовые воздействия пропорционально эффективной массе тонов колебаний. Эти усилия оказались примерно одинаковыми для всех моделей и далеки от реальных, по- 
скольку они определяются преимущественно одним осциллятором с эффективной массой $\sim 75 \%$ (см. рис.5). Этот график указывает на некорректность задания обобщенных сил на осцилляторы пропорционально их массам.

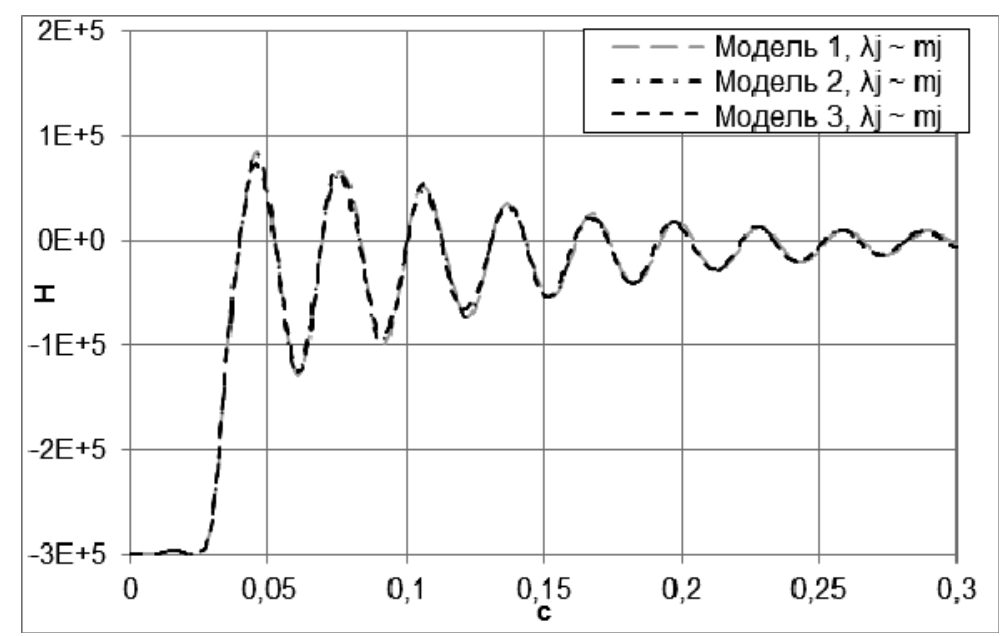

Рис.5. Усилия в заделке, определенные по КЭ-моделям бака с механическими аналогами, у которых $\lambda_{j}$ пропорционально $m_{j}$.

\section{4. Заключение}

Предложенная методика позволяет задать внешние силовые воздействия на механические аналоги таким образом, чтобы воздействие на систему, содержащую эти аналоги, было эквивалентно воздействию на реальную конструкцию. При этом должны выполняться два критерия:

- сходимость масс эквивалентных осцилляторов к общей массе топливного бака;

- сходимость суммы приложенных к осцилляторам сил к физической силе, а именно к тяге ДУ.

Таким образом, исследованы особенности построения механических аналогов, моделирующих продольные гидроупругие колебания баков с топливом, с использованием метода конечных элементов.

Разработанная методика учета активных сил может применяться для корректного задания внешних воздействий на конструкцию, моделируемую с использованием механических аналогов. Это может быть особенно важно при расчетном анализе нагрузок в процессе ОСИ двигателей, в том числе для оценки градиентов набора и спада тяги ДУ по динамической реакции конструкции огневого стенда и испытываемого объекта, а также при анализе нагрузок на перспективные многоразовые ступени $\mathrm{PH}$. 


\section{СПИСОК ЛИТЕРАТУРЫ}

1. Лиходед А.И. Об использовании механических аналогов при расчете колебаний оболочек, состыкованных со стержнями // Прикладная механика, 1978, т.14, №6, с.16-25; англ. пер.: Likhoded A.I. Using mechanical analogs in calculating vibrations of shells joined to rods // Soviet Applied Mechanics, 1978, v.14, №6, p.565-573.

2. Лиходед А.И. Построение механических аналогов для оболочек, последовательно включаемых в стержневую систему // Прикладная механика, 1986, т.22, №9;

англ. пер.: Likhoded A.I. Construction of mechanical analogs of shells serially connected into a rod system // Soviet Applied Mechanics, 1986, v.22, №9, p.871-877.

3. Chatman Y., Gangadharan S., Ristow J., Sudermann J., Walker C., Hubert C. Modeling and parameter estimation of spacecraft fuel slosh with diaphragms using pendulum analogs // Advances in the Astronautical Sciences, 2007, v.128, p.53-71.

4. Park K., Lee S., Shin S. Longitudinal characteristics analysis of a space launch vehicle using one-and three-dimensional combined modeling for pogo prediction // AIAA Space and Astronautics Forum and Exposition, 2018, Article № AIAA 2018-5416, 14 p.

5. Микишев Г.Н., Рабинович Б.И. Динамика твердого тела с полостями, частично заполненными жидкостью. - М.: Машиностроение, 1968, 531 с.;

Mikishev G.N., Rabinovich B.I. Dinamika tverdogo tela s polostiami, chastichno zapolnennymi zhidkostiu. - M.: Mashinostroenie, 1968, $531 \mathrm{s.}$

6. Колесников К.С. Динамика ракет. - М.: Машиностроение, 2003, 520 с.;

Kolesnikov K.S. Dinamika raket. - M.: Mashinostroenie, 2003, 520 s.

7. Кармишин А.В., Лиходед А.И., Паничкин Н.Г., Сухинин С.Н. Основы отработки прочности ракетно-космических конструкций. - М.: Машиностроение, 2007, 480 с.;

Karmishin A.V., Likhoded A.I., Panichkin N.G., Sukhinin S.N. Osnovy otrabotki prochnosti raketno-kosmicheskikh konstruktsii. - M.: Mashinostroenie, 2007, $480 \mathrm{~s}$.

8. Конюхов А.С. Имитационная динамическая модель жидкостных ракет-носителей пакетной компоновки // Проблемы прочности, 2015, №2, с.138-147;

англ. пер.: Konyukhov A.S. Dynamic simulation model of liquid-propellant packet scheme launch vehicles // Strength of Materials, 2015, v.47, №2, p.332-340.

9. Бужинский B.A. Динамика и устойчивость движения ракет. - Королев: Изд. ФГУП ЦНИИмаш, 2017, 270 с.;

Buzhinskii V.A. Dinamika i ustoichivost dvizheniia raket. - Korolev: Izd. FGUP TSNIImash, $270 \mathrm{~s}$.

10. Шмаков В.П. Избранные работы по гидроупругости и динамике упругих конструкций. - М.: Издательство МГТУ им. Н.Э. Баумана, 2011, 287 с.;

Shmakov V.P. Izbrannye raboty po gidrouprugosti i dinamike uprugikh konstruktsiy. - M.: Izdatelstvo MGTU im. N.E. Baumana, 2011, $287 \mathrm{s.}$

11. Григорьев В.Г. Методика расчёта параметров динамической модели упругой конструкции, содержащей жидкость // Избранные проблемы прикладной механики и математики. - М.: МАМИ, 2003, с.93-126;

Grigoriev V.G. Metodika rascheta parametrov dinamicheskoy modeli uprugoi konstruktsii, soderzhashchei zhidkost // Izbrannye problem prikladnoy mekhaniki i matematiki. - M.: MAMI, 2003, s.93-126.

12. Chiba M., Watanabe H., Bauer H.F. Hydroelastic coupled vibrations in a cylindrical con- 
tainer with a membrane bottom, containing liquid with surface tension // Journal of Sound and Vibrations, 2002, v.251, №4, p.717-740.

13. Бужинский B.A. Уравнения возмущенного движения ракеты как тонкостенной конструкции с жидкостью // ПММ, 2009, т.73, №6, с.959-964; англ. пер.: Buzhinskii V.A. The equations of the perturbed motion of a rocket as a thinwalled structure with a liquid // Journal of Applied Mathematics and Mechanics, 2009, v.73, №6, p.692-695.

14. Вин Ко Ко, Темнов А.Н. Колебания дискретно-стратифицированных жидкостей в цилиндрическом сосуде и их механические аналоги // Вестник МГТУ им. Н.Э. Баумана. Серия «Естественные науки», 2016, №3, с.57-69;

англ. пер.: Win K.K., Temnov A.N. Oscillations of Immiscible Liquids in a Stationary Cylindrical Vessel and their Mechanical Analogs // Herald of the Bauman Moscow State Technical University, Series Natural Sciences, 2016, №3, p.57-69.

15. Chiba M., Magata H. Influence of liquid sloshing on dynamics of flexible space structures // Journal of Sound and Vibrations, 2017, v.401, p.1-22.

16. Ma C., Zhang X., Luo Y., Zhang S. Dynamic behaviors of flexible fluid containers with fuel consumption // Proceedings of the Institution of Mechanical Engineers, Part C: Journal of Mechanical Engineering Science, 2015, v.229, №15, p.2737-2749.

17. Hao Y., Tang G.-A., Xu D., Yang $Q$. Finite-element modeling and frequency-domain analysis of liquid-propulsion launch vehicle // AIAA Journal, 2015, v.52, №11, p.3297-3304.

18. Lee S., Sim J., Shin S., Kim Y. Longitudinal modal analysis of a LOX-filled tank using the virtual mass method // Intern. J. of Aeronautical \& Space Sci., 2017, v.18, №4, p.807-815.

19. Бабаков И.М. Теория колебаний. - М.: Наука, 1968, 560 с.; Babakov I.M. Teoriia kolebaniy. - M.: Nauka, 1968, $560 \mathrm{~s}$.

20. Бондаренко А.Ю., Лиходед А.И., Малинин А.А., Сидоров В.В. Исследование вибродинамики конструкций при кинематическом и силовом внешнем воздействиях // Космонавтика и ракетостроение, 2017, №3(96), с.5-13;

Bondarenko A.IU., Likhoded A.I., Malinin A.A., Sidorov V.V. Issledovanie vibrodinamiki konstruktsiy pri kinematicheskom i silovom vneshnem vozdeistviiax // Kosmonavtika i raketstoenie, 2017, №3(96), s.5-13.

21. MD Nastran. Dynamic Analysis User's Guide. Chapter 11 - Coupled Fluid-Structure Analysis. Revision 0, June 25, 2010, 556 p.

22. Бондаренко А.Ю., Борисов М.А. Расчёт эквивалентных осцилляторов, имитирующих колебания жидкости в оболочках, с использованием метода виртуальных масс // 7-я Международная науч.-техн. конференция «К.Э. Циолковский - 160 лет со дня рождения. Космонавтика, радиоэлектроника, геоинформатика», Рязань, 2017, с.111-116; Bondarenko A.IU., Borisov M.A. Raschet ekvivalentnyx ostsilliatorov, imitiruiushchikh kolebaniia zhidkosti v obolochkakh, s ispolzovaniem metoda virtualnykh mass // 7-ia Mezhdunarodnaia nauch.-tekhn. konferentsiia «K.E. Tsiolkovsiy - 160 let so dnia rozhdeniia. Kosmonavtika, radioelektronika, geoinformatika», Riazan, 2017, s.111-116.

Поступила в редакцию 30.10.2019

После доработки 30.10.2019

Принята к публикации 23.12.2019 